\title{
Estrategia de comunicación organizacional en redes sociales: análisis electoral de la extrema derecha francesa en Twitter
}

\author{
Organizational communication strategies on social \\ networks: analysis of the French far-right's electoral \\ campaigns on Twitter
}

\author{
Uxía Carral; Jorge Tuñón-Navarro
}

Cómo citar este artículo:

Carral, Uxía; Tuñón-Navarro, Jorge (2020). “Estrategia de comunicación organizacional en redes sociales: análisis electoral de la extrema derecha francesa en Twitter". Profesional de la información, v. 29, n. 6, e290608. https://doi.org/10.3145/epi.2020.nov.08

Artículo recibido el 07-11-2019 Aceptación definitiva: 25-06-2020

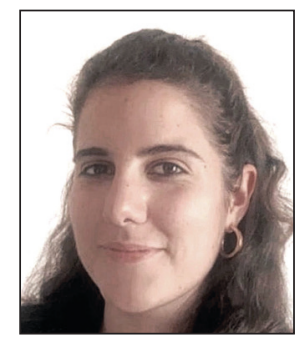

\author{
Uxía Carral $\bowtie$ \\ https://orcid.org/0000-0002-2329-3331 \\ Universidad Carlos III de Madrid \\ Departamento de Comunicación \\ Madrid, 126 \\ 28903 Getafe (Madrid), España \\ ucarral@hum.uc3m.es
}

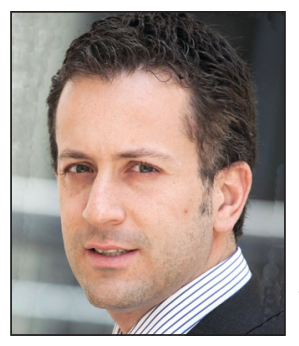

Jorge Tuñón-Navarro

https://orcid.org/0000-0003-0393-6560

Universidad Carlos III de Madrid

Departamento de Comunicación

Madrid, 126

28903 Getafe (Madrid), España

jtunon@hum.uc3m.es

\section{Resumen}

Desde las elecciones europeas de 2014, las organizaciones políticas de corte populista venían acrecentando las cuotas de poder que sus masas refrendaron en los comicios europeos de 2019. En Francia, el auge del Rassemblement National es en parte consecuencia de un cambio en la estrategia de comunicación de la organización. En el marco de su estrategia online pensada para las elecciones europeas, se analizaron los 1.256 tweets emitidos en su perfil oficial de Twitter durante los quince días de campaña electoral de 2014 y de 2019. Los resultados confirman la remodelación de su plan comunicativo online, lo que permitió a la formación crear un vínculo más estrecho con su audiencia para aumentar y fidelizar así el número de usuarios que podrían llegar a convertirse en sus potenciales votantes.

\section{Palabras clave}

Comunicación organizacional; Comunicación política; Campañas electorales; Campañas online; Redes sociales; Medios sociales; Populismos; Unión Europea; Rassemblement National; Twitter.

\section{Agradecimientos}

Este artículo forma parte de tres proyectos europeos en vigor financiados por la Agencia Europea de la Educación, la Cultura y el Audiovisual (EACEA) de la Comisión Europea, el módulo Jean Monnet Eucopol (referencia: 587167-EPP-1-2017-1-ES-EPPJMO-MODULE); la Red Jean Monnet

Cofinanciado por el programa Erasmus+ de la Unión Europea

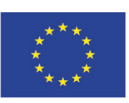
Openeudebate (referencia: 600465-EPP-1-2018-1-ES-EPPJMO-NETWORK); y la Cátedra Jean Monnet Eudfake (referencia: 610538-EPP-1-2019-1-ES-EPPJMO-CHAIR). El contenido de este artículo es responsabilidad exclusiva de los autores y la Eacea no es responsable del uso que pueda hacerse de la información aquí difundida. Asimismo, forma también parte del Proyecto Fakenews financiado por la Agencia Española de Investigación del Ministerio de Ciencia, Innovación y Universidades del Gobierno de España (Referencia: RTI2018-097709-B-I00) y del Proyecto de investigación I+D (Excelencia): Desafíos del proceso de construcción de un espacio europeo de derechos fundamentales (DER2017-83779-P). 


\begin{abstract}
Since the 2014 European elections, populist organizations have been gaining a share of power, as finally ratified by their voters in May 2019. In the case of France, the rise of National Rally (NR) is, partially, due to a change of its communication strategy. In particular, in its online strategy framework for the European elections, a total of 1,256 tweets published by its official Twitter account during the 15-day electoral campaigns in 2014 and 2019 have been analyzed. The results confirm a reconfiguration of its online communicative strategy and how this shift allowed the organization to create a closer link with its audience to increase and build loyalty among the number of users who could become $N R$ voters.
\end{abstract}

\title{
Keywords
}

Organizational communication; Political communication; Online campaigns; Electoral campaigns; Social networks; Social media; Populisms; European Union; Rassemblement National; National Rally; Twitter.

\section{Introducción}

El auge de los populismos, a la luz de los ejemplos de la elección de Donald Trump y del resultado del referéndum del Brexit, son una de las mayores preocupaciones europeas en la llamada era de la postverdad o la desinformación (Tuñón-Navarro; Bouza-García; Carral, 2019). A escala europea las organizaciones populistas son las que mejor se han sabido posicionar en las redes sociales, como centro de su estrategia de comunicación y relaciones públicas. Para dejar de ser minoritarias e incrementar su base de apoyo, son conscientes de la necesidad de planificar estrategias de comunicación organizacional transversales y necesariamente ancladas en las redes sociales (Schoeneborn, 2011). Ejemplo de ello es el Rassemblement National $(R N)$, partido de extrema derecha liderado por Marine Le Pen, cuya popularidad entre el electorado francés alcanzó su máximo histórico en las últimas elecciones en 2019. Tras su rotunda victoria en 2014, la formación consiguió revalidar el honorífico título de primera fuerza política de Francia en los comicios europeos el 26 de mayo de 2019, batiendo incluso a la opción centrista Renaissance, el partido del presidente francés Emmanuel Macron (Habermas, 2017; Bouza-García; Tuñón-Navarro, 2018). Este éxito se debe principalmente al cambio de imagen y relato que la organización política ha conseguido mostrar a las audiencias a través de una evolución en su plan comunicativo. Específicamente, la formación -dada la característica intrínseca a su calificación como populista- destaca por el buen uso de las redes sociales. Por ello sus prácticas discursivas en el mundo online configuran el objeto de estudio que ocupará esta investigación.

Actualmente resulta esencial para las relaciones públicas de las organizaciones hacer uso de las metodologías tecnológicas emergentes, especialmente online y a través de las redes sociales. Mientras varios académicos han analizado el discurso político sobre los nuevos medios y las redes sociales como Twitter (Eom et al., 2015; Kreiss, 2016b, Larsson; Kalsnes, 2014), en este trabajo tratamos de transferir este análisis a una parte del plan de acción comunicativa de las organizaciones políticas en campaña electoral, tomando como caso de análisis al partido por antonomasia de la extrema derecha francesa. En la actualidad las fórmulas online resultan cruciales para los actores políticos e institucionales que aspiran a comprometer a los ciudadanos e involucrar a las audiencias (Campos-Domínguez, 2017; López-Meri; Marcos-García; Casero-Ripollés, 2017), ya sea a nivel supranacional como en el caso de las estrategias de comunicación de la UE (Papagianneas, 2017; Bouza-García; Tuñón-Navarro, 2018; Tuñón-Navarro, 2017), o a nivel nacional como pretende demostrar este trabajo.

\subsection{Comunicación organizacional y narrativas digitales}

Actualmente, las estrategias de una comunicación organizacional en materia de relaciones públicas y promoción de imagen por parte de las entidades políticas se encuentran en una encrucijada de diferentes enfoques metodológicos. En primer lugar es imprescindible precisar la aproximación con la que aludimos a ciertos conceptos claves. Por una parte, nos remitimos a la interpretación de Andrade sobre "comunicación organizacional", quien explica el término como

"un conjunto de técnicas y actividades que sirven para desarrollar una estrategia encaminada a facilitar y agilizar el flujo de mensajes que se dan entre sus miembros y entre la organización y los diferentes públicos (Andrade, 2005, pp. 15-16)".

En esta definición se distinguen ambos tipos de comunicación organizacional dependientes de la variable "audiencia" a la que, en este caso el $R N$, se quiere dirigir:

- la comunicación interna, basada en

"el mantenimiento de buenas relaciones con y entre sus miembros" (Andrade, 2005, p. 17)

- la comunicación externa, entendida como

"el conjunto de mensajes emitidos por la organización hacia sus diferentes públicos externos, encaminados a mantener o mejorar sus relaciones y a proyectar una imagen favorable" (Andrade, 2005, p. 17).

Por tanto, en esta segunda categoría el abanico de audiencias del $R N$ incluye no solamente a los potenciales votantes y/o simpatizantes, sino también a los medios de comunicación, rivales y aliados políticos e instituciones. 
En consecuencia, el grado de vínculo positivo que pueda establecer la organización política con sus públicos externos quedará supeditado al resultado de las diferentes acciones estratégicas coordinadas por el equipo de relaciones públicas (Grunig; Grunig; Dozier, 2003). En cuanto al marketing, el branding resulta una herramienta imprescindible para asentar la visión holística (valores, objetivos) y la imagen (Canel; Sanders, 2012) del RN. Ejemplo de ello ha sido el cambio de nombre del propio partido llevado a cabo en 2018. La sustitución de Frente por Agrupación induce a la audiencia a asociar el partido con valores de índole positiva como la inclusión frente a la discriminación. En relación con la comunicación, la clave de su engagement con las audiencias reside en las conductas (relatos, encuadres, apelaciones, etc.) que efectúa la propia organización política en torno a la narrativa, con la intención de influir en la opinión pública. Es en este último aspecto, la comunicación, donde se circunscribe el foco de este trabajo, y específicamente en la evolución de la comunicación online del $R N$.

La narrativa se ha convertido en un elemento clave de la comunicación organizacional online durante los últimos años (Maarek, 2014; D’Adamo; García-Beaudox, 2016; García-Orosa; Vázquez-Sande; López-García, 2017; Tuñón-Navarro; Carral, 2019; Tuñón-Navarro; Catalán-Matamoros, 2020). Asimismo, el desarrollo de las nuevas tecnologías de la información y la comunicación e internet (Serrano-Cobos, 2016), además de la innovación y la experimentación (Salaverría, 2015; Peñafiel, 2016), han vinculado definitivamente las narrativas digitales a la comunicación organizacional, evolucionando desde los primeros esfuerzos comunicativos eminentemente textuales hasta los más recientes, conceptualizados como multiplataforma (Guerrero-Pico; Scolari, 2016).

Apoyándose en las innovaciones derivadas de los cambios tecnológicos se han acuñado términos definitorios del uso de las nuevas aplicaciones tecnológicas en la comunicación, siendo uno de los más celebrados el de "tecnopolítica" (Gutiérrez-Rubí, 2014), que incide en la necesidad de nuevos lenguajes y narrativas. Profundizar en estos usos tecnopolíticos y evaluar su eficacia representa una prioridad, tanto a nivel profesional como académico (Gutiérrez-Rubí, 2014). Los departamentos de comunicación y marketing (principalmente de los partidos políticos) asumen hoy la necesidad de orquestar múltiples acciones y de hacerlas converger en la búsqueda de un mismo resultado (Larrondo-Ureta, 2016). Se ha podido verificar una clara evolución marcada por estrategias específicas para el medio con mayor poder de proyección, la Web (García-Orosa, 2009; Túñez-López, 2012), que progresivamente viene siendo acompañada y/o reemplazada por las pujantes redes sociales. Todo ello obliga a los departamentos de relaciones públicas a centrar cada vez más su foco de atención en la creación de planes de comunicación organizacional online omnicomprensivos.

Asimismo, la relevancia de las estrategias online ha surtido efecto en el ámbito de la investigación, implicando el traslado de varias técnicas clásicas a los modernos espacios comunicativos online (Fielding; Lee; Blank, 2008; Hine, 2005; Jones, 1998), como encuestas y entrevista online, observación participante y etnografía online, el análisis de redes sociales online o audiencias digitales, y el análisis de contenido web o análisis audiovisual, entre otras modalidades. Más allá de las conversacionales, una de las nuevas técnicas líderes en la investigación social online en los últimos años es la Ilamada etnografía digital o virtual (Fielding; Lee; Blanck, 2008; Hine, 2004; 2005; Kozinets, 2010). La etnografía virtual generalmente representa una forma simple de etnografía que trata el ciberespacio como un campo para observar la realidad etnográfica. Sin embargo, la tendencia más reciente implica la etnografía virtual exclusivamente dentro del campo online mediante modelos combinados o multi-ubicados que consideran la hibridación online-offline. A lo largo de este trabajo tratamos de reivindicar el uso de una técnica reformulada de un eminente análisis cuantitativo en el marco de la comunicación organizacional de carácter político. Ciertamente, la etnografía virtual en calidad de instrumento narrativo se puede entender como un método para enriquecer el conocimiento de los efectos y la influencia de los medios, así como captar no sólo los trabajos cotidianos de la comunicación política, en la producción y los fines de recepción (Spitulnik-Vidali; Peterson, 2012).

\subsection{Comunicación online y redes sociales}

La comunicación organizacional y el marketing político se han modernizado, y desde la campaña que en 2008 encumbró a Barack Obama a la Casa Blanca, no han podido prescindir de las redes sociales en general, y de Twitter en particular, como canales intermediadores del mensaje. Con gran éxito demostraron que pueden servir para proyectar un concepto preciso de un candidato (Jivkova-Semova; Requejo-Rey; Padilla Castillo, 2017), por lo que su influencia en el marco de la comunicación de las organizaciones políticas no ha hecho sino crecer en todas las democracias avanzadas (Bimber, 2014; López-Meri; Marcos-García; Casero-Ripollés, 2017).

Tal y como sucede desde hace una década con las campañas, los planes de comunicación de las organizaciones políticas ya no pueden entenderse sin la inclusión de las redes sociales. Estas se han constituido como un canal esencial de cualquier estrategia de comunicación que pretenda ser efectiva en la interactuación con las audiencias más jóvenes, debido a su capacidad de reducir la barrera psicológica y geográfica entre el usuario político y el seguidor. Concretamente, ni instituciones, ni corporaciones, ni organizaciones políticas, ni sus propios líderes han podido resistirse a las potencialidades derivadas de la red social más indicada para el debate y las estrategias de comunicación de las organizaciones políticas: Twitter. 
Resulta conveniente conocer más acerca de su utilización práctica por parte de partidos y líderes políticos. En ese sentido, existe ya un volumen considerable de investigación acerca de (Bouza; Tuñón-Navarro, 2018, p. 1240):

- extensión o frecuencia de su utilización;

- temáticas abordadas;

- motivos de su utilización preferente.

Sin embargo, se ha prestado menos atención a las funciones atribuidas a las plataformas como parte de las estrategias de comunicación de los partidos y de sus líderes (López-Meri; Marcos-García; Casero-Ripollés, 2017). A pesar de ello, a niveles supranacionales existe un cierto debate sobre el efecto de dichas redes para la comunicación de las organizaciones políticas paneuropeas (Barisione; Michailidou, 2017). Como se ha señalado, dicha red tiene potencial para reducir enormemente los costes de transacción y crear una nueva infraestructura también de comunicación organizacional. Es por ello que investigaciones académicas como la presente, pueden analizar las principales funciones atribuidas a Twitter en el marco de los discursos de las organizaciones políticas, a saber:

- su utilización en la configuración de las estrategias de relaciones públicas y comunicación;

- funciones atribuidas/esperadas a la red social por parte de las organizaciones;

- distribución del mensaje, su impacto y su engagement sobre las audiencias;

- autoría y personalización de las relaciones públicas a través de redes sociales y específicamente en el contexto de Twitter.

\subsection{Twitter en las relaciones públicas y la comunicación de las organizaciones políticas}

Durante la última década hemos asistido a la proliferación de las redes sociales como instrumento crucial para la formulación y promoción de ideas a través de un nuevo fenómeno que Scolari et al. (2012) denominan user-generated content, la creación y/o difusión de contenido online (texto, vídeos, imágenes) tanto por parte de los perfiles políticos como por sus fieles seguidores (Duggan, 2015; Quan-Haase; Sloan, 2016; Castillo-Esparcia; Guerra-Heredia; Almansa-Martínez, 2017). Si bien Twitter está lejos y no tiene previsión de destronar a Facebook, la red social con más usuarios a escala global (Galeano, 2019), sus características propias han convertido a Twitter en la red social preferida tanto para el debate y la comunicación de las organizaciones políticas, como para la propia investigación social y científica en este campo (Steward, 2017; Campos-Domínguez, 2017; López-Meri; Marcos-García; Casero-Ripollés, 2017).

Dentro del marco de la comunicación de las organizaciones políticas y las relaciones públicas, Twitter puede ser utilizada para diferentes funciones. Comenzó siendo analizada su aplicación en el marco de las campañas electorales, habiéndose verificado diferentes aproximaciones:

- comunicación estratégica de las organizaciones políticas y de sus candidatos (Parmelee; Bichard, 2012);

- información de campaña y diseminación de discurso (Jackson; Lilleker, 2011);

- fomento de la participación y movilización de las audiencias (Gainous; Wagner, 2014);

- promoción, imagen y auto-referencia en relación con la propia campaña (Jivkova-Semova; Requeijo-Rey; Padilla-Castillo, 2017).

No obstante, no todas las investigaciones han podido probar que la efectiva interacción entre las organizaciones políticas y sus audiencias predilectas tengan lugar (Alonso-Muñoz; Miquel-Segarra; Casero-Ripollés, 2016; Tuñón-Navarro; Carral, 2019; Tuñón-Navarro; Catalán-Matamoros, 2020). Incluso, otras investigaciones sostienen que la interacción se da más con los periodistas que con los ciudadanos (Graham; Jackson; Broersma, 2016), por ser Twitter la red social de más relevancia entre las organizaciones políticas y los medios de comunicación (Jivkova-Semova; Requeijo-Rey; Padilla-Castillo, 2017), ya que aporta un componente informativo que le confiere mucho valor en términos de estrategia política (Rodríguez-Andrés; Ureña-Uceda, 2011).

No podemos olvidar otro de los usos más frecuentemente verificados de Twitter, el de la personalización o enfoque del discurso en el individuo emisor muy por encima del contenido del mensaje emitido. Precisamente en algunos casos, los líderes de las organizaciones políticas, en el marco de su estrategia de comunicación, utilizan Twitter para compartir detalles de sus vidas privadas (Parmelee; Bichard, 2012; Veerger; Hermans; Sams, 2013), en un intento de humanizarse ante sus audiencias (Jackson; Lilleker, 2011; Bentivegna, 2015), incluso por medio de un tono informal o empleando el humor (Jivkova-Semova; Requeijo-Rey; Padilla-Castillo, 2017), en una práctica cuya extensión, frecuencia y eficacia depende también de la zona geográfica de aplicación (Scherpereel; Wohlgemuth; Schmelzinger, 2016; López-Meri; Marcos-García; Casero-Ripollés, 2017).

Además, hace tiempo que se está analizando el potencial derivado de la comunión de sinergias (hibridación) entre los medios digitales y los medios de comunicación tradicionales (Chadwick, 2013), o la falta de comunión de dichas sinergias (des-hibridación). En ese contexto las organizaciones políticas han visto en redes sociales como Twitter plataformas cruciales para sus estratégicas de relaciones públicas y comunicación para maximizar sus impactos sobre sus audiencias, motivo por el cual tratan de adaptar sus planificaciones tradicionales a las potencialidades ofrecidas por las redes sociales (Casero-Ripollés; Feenstra; Tormey, 2016).

El branding, las relaciones públicas, la reputación y la comunicación simétrica pueden constituir herramientas esenciales para la profundización en la comunicación 


\subsection{Estrategias de comunicación a través de Twitter}

No sólo se puede afirmar que las redes sociales han entrado a jugar un papel decisivo para las relaciones públicas de las organizaciones políticas, sino que la dependencia que los medios convencionales han adquirido sobre ellas ha provocado un cambio de dominio en el plano comunicativo al que Lévy (2004) denominó 'ciberdemocracia'. En efecto, la aparición de las tecnologías de la información y comunicación (TICS) ha otorgado a la comunidad de usuarios el poder decidir la prosperidad, popularidad y visibilidad de la conversación política (Mazzoleni, 2001). Los dirigentes todavía mantienen la preponderancia suficiente como para elegir qué temas introducir en la agenda y cuáles omitir, es más, incluso pueden filtrar información o difundir opiniones contrarias a lo expuesto en los medios (Aruguete, 2017), pero la notoriedad de su discurso depende ahora del nivel de implicación que los usuarios expresen con la causa (Carracedo, 2002).

En definitiva, a diferencia del escenario presencial o físico, es la sociedad conectada quien tiene el poder de otorgar un largo o efímero camino al devenir político (Mosca; Vaccari, 2011), lo que está implicando una reestructuración del sistema comunicativo de la política (Chadwick, 2006). Así, la omnipresencia de Twitter en la agenda diaria ha creado por otra parte a las organizaciones la necesidad de estar presentes y de actualizarse, con lo que sus comportamientos digitales también inciden sobre sus actuaciones y pautas dialécticas que se mantienen en la vida real (Elías, 2015). Además, la propia adaptación de las estrategias de comunicación de dichos actores a este medio indica su relevancia, especialmente durante los períodos de planificación y campaña (Maarek, 2011; Bruns; Burgess, 2011, Vergeer; Hermans, 2013; Jungherr; Schoen; Jürgens, 2015; Tuñón-Navarro; Catalán-Matamoros, 2020).

Más allá de la ya argumentada idoneidad de Twitter para las relaciones públicas y la comunicación de las organizaciones políticas, una gestión activa y regular en sus cuentas propicia la dinamización del diálogo, la difusión del mensaje y el incremento en popularidad del perfil político (Larsson, 2015). Además, su interactividad mediante las menciones y los hashtags da lugar a hilos de conversación que indirectamente promueven

"la creación de vínculos entre los diversos usuarios" (Larsson, 2015, p. 89),

a partir de los cuales se pueden acabar formando círculos de apoyo. Asimismo, la participación -en forma de retweets (RTs), "me gustas" (favs) y comentarios- ayuda a reforzar la atención hacia la cuenta de organización, otorgándole una mayor visibilidad con el fin de que se traduzca en un incremento de seguidores y, por tanto, en mayor influencia (Sanjuán-Santonja, 2015).

\section{Todo parecería indicar por tanto que}

"las reglas del juego han cambiado y ahora el concepto clave es la conversación. El votante ya no sólo escucha, sino que también habla" (Rodríguez-Andrés; Ureña-Uceda, 2011, p. 31).

Sin embargo, multitud de autores ponen en duda las palabras de estos autores, al manifestar que en realidad los actores políticos no aprovechan al máximo los recursos que les facilitan las redes sociales (Golbeck; Grimes; Rogers, 2010; Enli; Skogerbø, 2013; Adams; McCorkindale, 2013, Kreiss, 2016a; Gainous; Wagner, 2014). Aunque ponen la semilla del debate con la publicación de un primer tweet, después no entablan conversación con los usuarios, ni siquiera con los perfiles afines, con lo que la utilización que hacen de las redes no potencia el fin último de estas, que es la comunicación bidireccional.

En materia de comunicación organizacional se ha advertido mayormente la utilización de Twitter:

- como mero canal de autopromoción y tal parece comenzar a ser su eficacia, que este cometido se ve aún más explotado durante el período de las campañas (Jungher, 2014);

- para movilizar a los usuarios y para animarlos a participar (Gainous; Wagner, 2014);

- como un anzuelo con el fin de capturar la atención de los medios convencionales y centrar la cobertura en su figura o influir en sus publicaciones mediante la queja o la promoción en sus redes de sus intervenciones (Stromer-Galley, 2014).

De esta manera, la aceptación de las redes sociales (y de Twitter en particular) como un canal más por el que transmitir su mensaje ha dado lugar a un plan bicéfalo en la estrategia de relaciones públicas y de comunicación de las organizaciones políticas. Ahora ya se mueven en un "entorno híbrido", a caballo entre los medios tradicionales y las redes, por lo que sus dinámicas de planificación se han reformulado para así obtener el máximo provecho de ambos (Casero-Ripollés; Feenstra; Tormey, 2016).

\section{Metodología}

La finalidad de esta investigación es demostrar una variación en el modo de relacionarse públicamente del Rassemblement National $(R N)$ a través de Twitter, considerado en la actualidad como un elemento con "poder y valor" dentro de las "relaciones públicas" y la "autocomunicación de masas" (Castells, 2009, p. 68). La demostración de este propósito sumaría una evidencia más al cúmulo de las descritas por diversos autores (Fernández-Vázquez, 2019; Betz, 2017; Crépon; Dézé; Mayer, 2015) con el fin de afirmar que existe un blanqueamiento de imagen del RN desde la llegada de Marine Le Pen en 2011. Por lo tanto, la hipótesis principal de nuestro trabajo incide en el cambio de estrategia de comunicación online del $R N$ con el fin de ampliar su potencial abanico de votantes. Para ello el primer propósito va encaminado a demostrar el afianzamiento de Twitter como canal de comunicación de las organizaciones políticas; y el segundo, a relacionar el incremento del número de autores con el engagement en sus mensajes. 
La consecución de estos dos objetivos demostraría una tendencia emergente en la comunicación organizacional de los partidos políticos que Eom et al. (2015) explican como la modelización de dinámicas de la atención/participación colectiva por parte de los usuarios en las redes sociales. Por ende, las preguntas de investigación son las siguientes:

1. ¿El $R N$ valora su presencia en Twitter como un recurso principal de su estrategia de comunicación?

2. ¿Ha supuesto la apertura en su autoría una mayor viralidad de su mensaje?

Por una parte, en cuanto al período temporal analizado, se ha pretendido hacer una comparativa en el apogeo del partido tras su regeneración, lo que suponía estudiar al $R N$ a partir del nombramiento de Marine Le Pen como líder (16/1/2011). Por otra parte, hay que tener en cuenta las características del sistema electoral de Francia, que consta de dos vueltas. Este factor impide un tratamiento igualitario de los datos entre todos los comicios. En consecuencia, cribando las doce veces que los franceses han ido a las urnas desde 2011, los resultados perfectamente comparables con mejor porcentaje para el $R N$ se dieron en las elecciones al Parlamento Europeo. De modo que el objeto de estudio quedó enmarcado en torno a ambas campañas electorales al PE de 2014 y 2019 (9-24/5/2014 y 10-25/5/2019).

Ante este espacio de conversación tan plural, se decidió sistematizar el escenario mediante la utilización de una metodología cuantitativa híbrida compuesta por procedimientos computacionales y manuales. El análisis de contenido es uno de los métodos más útiles para el estudio de campañas electorales, ya que se trata de una técnica objetiva que permite la descripción, pero también la interpretación y la generalización de patrones (Eiroa; Barranquero, 2017). De esta manera, el corpus está conformado por 1.256 tweets emitidos por el perfil oficial del RN (@RNational_off), de los cuales, 254 publicaciones corresponden al ejercicio de 2014, y, 1.002, al período de 2019.

Con la aplicación Twlets, gratuita y online, se han recopilado los tweets y se han analizado, para lo cual se ha empleado una ficha de elaboración propia (tabla 1, Anexo), inspirada en investigaciones anteriores (Congosto, 2015; Calvo; Renedo-Farpón; Díez-Garrido, 2017; Marcos-García, 2018). De modo que se elaboró un sistema de ocho variables ordenadas en tres niveles. En este sentido, se debe subrayar que han sido necesario establecer "intervalos de prorrateo" siguiendo las recomendaciones de anteriores investigaciones sobre engagement (Giglietto; Selva, 2014). Esta decisión se debe a la disparidad de resultados inducidos en una primera revisión previa al comienzo del análisis y al deseo de evitar posibles desviaciones y sesgos. Posteriormente, dada la complejidad que supondría calcular manualmente tal cantidad de tweets, los datos codificados fueron introducidos en la base de datos del programa IBM SPSS (v. 25), con el cual se han cruzado las variables para obtener los porcentajes y correlaciones que integran el núcleo de este análisis de contenido y que se proceden a presentar a continuación.

\section{Resultados y discusión}

\subsection{H1: El $R \boldsymbol{N}$ valora su presencia en Twitter como un recurso principal de su estrategia de comunicación}

En una primera lectura superficial de los resultados ya se podía observar un cambio significativo en la estrategia del $R N$ : un incremento del $400 \%$ en el número de tweets publicados o retweeteados por el propio partido en su cuenta oficial de Twitter, pasando de 254 mensajes en 2014 a 1.002 emitidos en la campaña electoral de 2019. Si bien en 2019 el uso de Twitter está mucho más extendido que en 2014, esta cuadruplicación de los datos indica también un mayor esfuerzo del partido por implicarse en la comunicación online con los ciudadanos. No obstante, no sólo se tweeteó más, sino también mejor.

El cambio la procedencia de contenido agregado señala la primera gran transformación en la estrategia llevada a cabo entre estos cinco años. En 2014, el 98\% de los enlaces dirigían al usuario a la propia web, porque encauzando el mensaje por una sola vía de comunicación se reducía el riesgo de tergiversación del relato. No obstante, el manejo de las redes sociales era muy rústico y monótono, los textos visibles en el propio muro eran escuetos y la apariencia de las publicaciones poco atractiva. La comunicación se producía en una sola dirección (partido a seguidores) y el ejercicio resultaba incómodo para el usuario, pues el estilo de publicación obligaba a los lectores a migrar de la red social hacia la web para seguir profundizando en el contenido. Todo ello condujo a un engagement de la audiencia muy bajo o en ocasiones nulo.

Sin embargo, su comunicación en redes sociales fue mejorando hasta conseguir elaborar una campaña electoral 2.0 para las europeas de 2019.

Se produjo una reducción drástica (-92\%) de los tweets que contenían un enlace a la web, lo que confirma que desde el partido se propusieron un cambio de estrategia. En primer lugar, se ha demostrado que una apertura del mensaje en las redes no implica su heterogeneidad, pues el $R N$ sigue manteniendo el control sobre lo que se publica (e incluso la posibilidad de editarlo según sus intereses). También, $R N$ ha entendido que el contenido insertado en el propio tweet provoca un aumento en la potencial expansión de su mensaje, un elemento que además ayudaría a acrecentar los círculos de apoyo. Por consiguiente, la campaña electoral de 2019 tuvo un mayor dinamismo en la comunicación, reflejado en que el $86,8 \%$ de la muestra de tweets iba acompañada principalmente de fotografías $(44,3 \%)$ y vídeos/audios (33,9\%) (gráfico 1 ).

El modelo de relaciones públicas diseñado ha permitido al Rassemblement National virar la reputación fascista del partido hacia la de protector de la soberanía y los intereses nacionales 
En segundo lugar, la comunicación de la organización también varió respecto a los tweets publicados por el $R N$ con enlaces directos a noticias procedentes del propio portal web de los medios de comunicación. El poco o nulo foco mediático que recibía el partido ultraderechista en 2014 pretendía así ser contestado proporcionalmente en sus comunicaciones. En 2014, como los medios no contemplaban la aparición del $R N$ en sus programas y crónicas, el partido tampoco incluía en sus publicaciones links que redireccionaran a sus seguidores a webs de los medios. No obstante, como las expectativas previas a las últimas elecciones eran altas, la relación con los medios también cambió. Actualmente el partido populista no pretende buscar aliados en los medios de comunicación, pero es consciente de que necesita estar presente en ellos, por lo tanto durante la campaña de 2019 se ha visto forzado a interactuar de manera más frecuente.

Por ello se generalizó la aparición de pequeños clips de vídeo de sus intervenciones en programas de radio y de televisión, consiguiendo que uno de cada tres tweets publicados incidiera sobre esa promoción futura o pasada de los diferentes integrantes de la organización en medios. Asimismo el $R N$ abrió tímidamente la puerta, y de forma paradójica mediante las redes sociales, a una relación más estrecha con los medios tradicionales. En un gesto sin precedentes en la comunicación del $R N$, en la campaña electoral de 2019 , se difundió una serie de enlaces de noticias provenientes de medios en un $1,4 \%$ de su muestra de tweets.

Aunque el porcentaje es extremadamente bajo, ya que tan sólo equivale a 14 tweets de un total de 1.002, se debe hacer una lectura más profunda. El avance del $R N$ en la difusión de su mensaje ha sido galopante en los últimos años y así lo han demostrado los resultados de las urnas. Ha transitado desde 2014, cuando se temía una contaminación de su mensaje si se exteriorizaba en su propio perfil oficial de Twitter, hasta 2019, momento en el que tweeteó de media más de 50 mensajes diarios, modernizó su contenido hacia formatos audiovisuales, e incluso animó a sus seguidores a leer lo que los medios decían de ellos. Si bien hay que admitir que los enlaces compartidos fueron elegidos porque se adecuaban al relato que convenía al $R N$ para las elecciones europeas, dejar entrar a un "no-aliado" en su espacio comunicativo siempre supone un cierto riesgo, que la organización decidió correr esperando poder beneficiarse en las urnas.

Por último, una tercera vía de cambio se percibe en el surgimiento de pequeñas conversaciones, ausentes por completo en 2014 y, que en 2019 nacieron motivadas por dos técnicas:

- una de ellas se produce al responder mediante el retweet con comentario superior, ejercicio que el $R N$ ejecutó para el $14,6 \%$ de la muestra en 2019;

- apelación directa a un sujeto a través de la mención (gráfico 2) en el propio tweet que publica el partido galo. En este sentido, cinco años atrás, los mensajes sin apelaciones constituían el modelo de comunicación del $R N$ en un $80 \%$, en cambio hoy en día sólo cubren un tercio de las publicaciones.

Sin embargo, aunque puede parecer que el $R N$ ha optado por introducir una comunicación bidireccional en su estrategia, en ningún momento ha llegado a crear un hilo de conversación en Twitter con otro actor político. Las menciones que realizan -de manera errónea, aunque puede que esto también sea parte de su técnica- son sólo un cebo de provocación que se expone delante de la audiencia. Este tipo de formaciones aspira a una guerra de representaciones y no a un debate; por ende, no interesa tanto un enfrentamiento online con sus rivales, si no un espacio donde dar a conocer a sus seguidores la figura de sus enemigos ("los otros"), según la descripción en 140 caracteres que beneficia al $R N$.

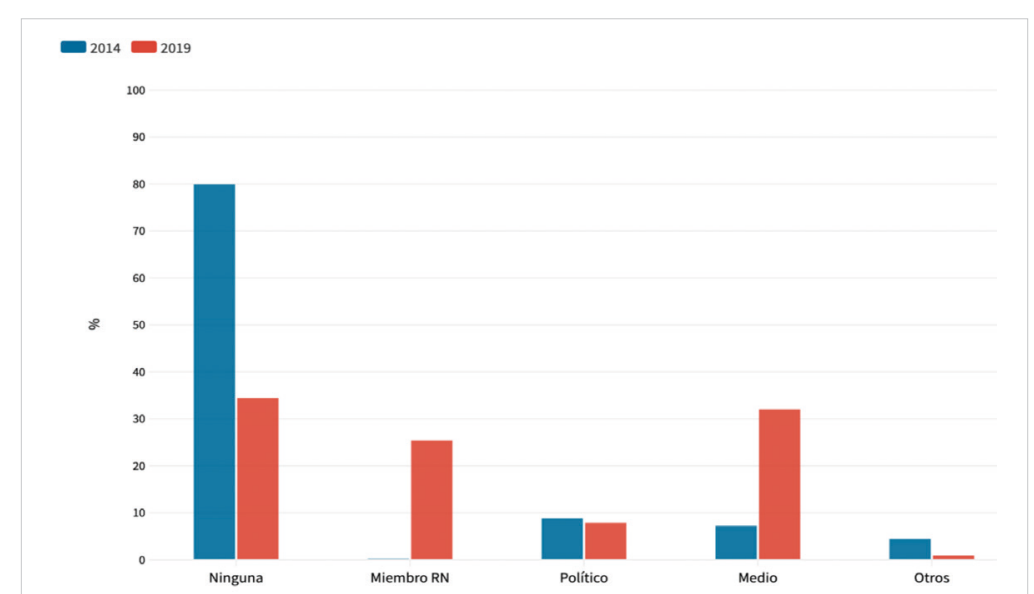

Gráfico 2. Evolución de la distribución en la participación del RN (2014-2019) 


\subsection{H2: La apertura en la autoría del $R N$ ha supuesto una mayor viralidad de su mensaje}

El partido de Le Pen venía de conseguir en 2014 los mejores resultados de su historia en unas elecciones europeas y de situarse por primera vez como principal organización política en Francia. Por lo tanto, el objetivo para 2019 consistía en aumentar la diferencia con el resto de las formaciones o, en el peor de los casos, no perder esa primera posición. Para ello no bastaba con asegurar el voto de los reductos más radicales y populistas, sino que hacerse con una mayoría en las urnas exigía promover el discurso del $R N$ entre un electorado más plural, y por tanto un cambio de estrategia de comunicación.

El hecho de renombrarse como "Agrupación" (Rassemblement) implica la obligación de un mayor pluralismo en las trincheras y no tanto de un líder que capitanee a las masas. Por eso, el $R N$ expandió el número de voces autorizadas para proclamar el mensaje del partido pero además les otorgó especial visibilidad en el perfil oficial. En el gráfico se aprecia la diferencia entre el período electoral de 2014, donde la totalidad de las publicaciones emitidas durante esos quince días provenían del propio @RNational_off, y la campaña de 2019, en la que solamente el 40\% de todos los mensajes fueron escritos por la cuenta. Esta disparidad provoca que seis de cada diez tweets aparecidos en el muro del $R N$ sean retweets a otros miembros del partido.

Dentro de estos, la primera figura a quien se va a dar espacio es indiscutiblemente a la presidenta, Marine Le Pen, que acapara un $21,4 \%$ de la muestra emitida en campaña. Seguidamente se posiciona Jordan Bardella, con un porcentaje bastante similar, un 20,8\%, pues en una campaña europea el cabeza de lista debe funcionar como líder. Este sostén se realiza con la intención de crear una imagen pública favorable a los líderes (Castillo-Esparcia; Smolak-Lozano; Fernández-Souto, 2017). A partir de ahí, el peso de la jerarquía se impone y el resto de los miembros del partido tienen un grado de visibilidad menor. Todos ellos consiguen acaparar un $17 \%$ de los tweets aparecidos en el muro del partido durante esos quince días; no obstante, su valor como autores será proporcional a la dimensión de sus apariciones.

El crecimiento de los miembros del $R N$ no sólo se ha dado en la autoría, también en las menciones, como ya explicamos en el gráfico 2. En consecuencia, el mensaje que reciben los seguidores del $R N$ procede de múltiples personalidades, con lo que se ensanchan las posibilidades de conectar con diversos prototipos de usuarios y buscar su voto desde la posición con la que más se reconozcan (la presidenta, el líder europeo, el alcalde local, etc.). En cambio, apenas se deja espacio a agentes que pudiesen tergiversar su mensaje. Tan sólo un 0,3\% de la muestra de 1.002 tweets recogidos en 2019 procedían de rivales políticos y un $0,1 \%$ eran retweets a medios de comunicación.

Muestra de la nueva estrategia del $R N$ de hilvanar un relato a través de una multiplicidad de actores es el nivel de engagement comparado entre 2014 y 2019. En 2014, cuando la autoría de los tweets permanecía totalmente bajo las directrices de la cuenta oficial, la media de RTs más frecuente (en el 57,9\% de los casos) se encontraba en el intervalo de seis a veinticinco retweets de los mensajes del $R N$ por parte de los seguidores. En cambio, tras la repartición del espacio público de la cuenta del $R N$ para 2019, el vínculo de los seguidores del $R N$ con los mensajes tweeteados se ha fortalecido, ya que los usuarios suelen retweetear de veintiséis a cien veces los mensajes -como se observa en el tercer intervalo del gráfico 4-. Además, si profundizamos en los datos obtenidos de la muestra de 2019 (gráfico 4), observamos que existe un punto de inflexión a partir del cuarto intervalo (101-200 RTs). Los tweets publicados-cada círculo equivale a un tweet- por la propia cuenta del partido (azul oscuro) constituyen una mayor parte de la muestra, pero consiguen un alcance en número de retweets inferior, predominando únicamente en los tres primeros rangos. En cambio, aquellos mensajes publicados por los demás autores y retweeteados por el perfil del $R N$, suponen una menor cantidad, pero tienen en los intervalos superiores un radio de alcance más alto que los de la propia cuenta. Así se puede ver en el caso de Marine Le Pen (rojo) y Jordan Bardella (amarillo), cuyos mensajes, por ejemplo, es más probable que lleguen a recibir más de 500 RTs que los del propio $R N$.

Atendiendo a la implicación de la audiencia en forma de "me gusta", se puede apreciar un comportamiento similar, de tendencia positiva, incluso con unos resultados todavía más definitorios. A diferencia de 2014, cuando ocho de cada diez tweets no superaron la barrera de los cinco favoritos y ningún mensaje pudo rebasar los veinticinco "me gusta", en 2019 la horquilla más frecuente $(36,4 \%)$ de "me gusta" otorgados por parte de los usuarios a los mensajes del $R N$ recogió entre veintiséis y cien favs, y hasta un $18,7 \%$ de los tweets alcanzó la barrera psicológica de los más de 500" favoritos.

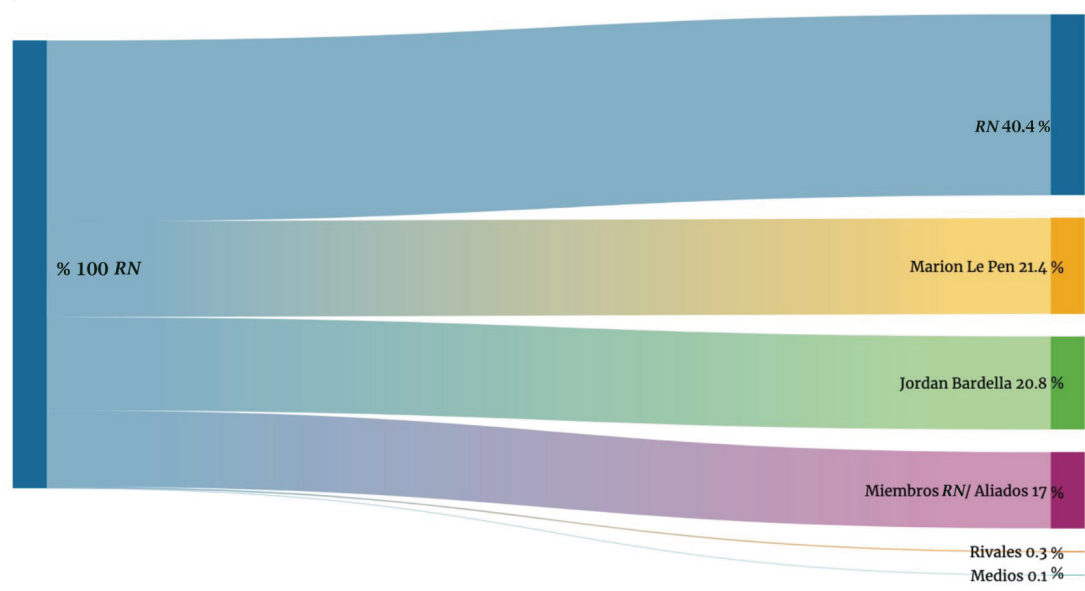

Gráfico 3. Evolución en la autoría del RN (2014-2019) 
Concretamente, en la medición del número de "me gusta" de cada tweet en relación con su autoría (gráfico 5), se aprecia todavía mejor la fructífera aportación de los nuevos autores al radio de alcance que pueden conseguir las publicaciones del $R N$. Por una parte, se percibe una marcada diferencia en el apoyo que reciben los tweets por el partido populista (azul oscuro), situados principalmente en intervalos más bajos (1, 2 y 3 ), y los retweets del conjunto -Marine Le Pen (rojo), Jordan Bardella (amariIlo) y otros miembros del partido (naranja)-, que han aumentado en cantidad y en la calidad del engagement, dominando de forma evidente los intervalos superiores. Por otra parte, cabe destacar la viralidad que consigue Marine Le Pen (rojo) con sus publicaciones, siendo el emisor cuyos mensajes reciben más favoritos en todas las horquillas superiores a doscientos "me gusta". En especial, se debe subrayar la popularidad que han otorgado a la cuenta del partido tanto la líder como su número uno europeo, Jordan Bardella, ya que gracias a los mensajes de ambos (rojo y amarillo respectivamente), esos tweets recibieron más de 500 favoritos.

La última evidencia del cambio de estrategia del $R N$ en cuanto a autoría se resuelve con la comparación del feedback escrito obtenido en ambas campañas electorales. En primer lugar, se debe resaltar la reducción en el número de publicaciones en el perfil del $R N$ que no recibieron ninguna contestación por parte de la audiencia, pasando de un $57,1 \%$ en el 2014 a un $13,87 \%$ en 2019. Esto significa que la diversidad de actores ha provocado una mejora de la retroalimentación de la audiencia aproximadamente en el $75 \%$ de la muestra. No obstante, en esta ocasión la demostración de nuestra hipótesis se deduce preferiblemente parangonando entre los datos de 2019 sobre autores individuales.

Como se observa en el gráfico 6, podemos señalar al perfil de la formación populista como el autor con mayor número de tweets publicados, pero a su vez con el grado de respuesta más bajo (violeta) y sin llegar a alcanzar ningún tweet las doscientas contestaciones. Seguidamente nos encontramos con dos perfiles bastante semejantes a la hora de medir su feedback en la campaña de 2019. Tanto Le Pen como Bardella publicaron cuantitativamente de forma similar, cada uno de ellos alrededor de un $20 \%$ de la totalidad de los mensajes recogidos de la cuenta del $R N$, aunque la notoriedad de la líder hizo que sus mensajes lograsen mejores resultados en los intervalos superiores. Así se puede observar en la 
circunferencia de Marine Le Pen, que cuenta con más número de mensajes contestados por 300 o más veces (morado y verdes) que la circunferencia de Bardella. No obstante, la elaboración de un análisis de recepción de los comentarios ayudaría a una mejor comprensión de esta dinámica, pues en esta investigación no se ha podido especificar si el sentimiento de estos fue positivo o negativo.
El $R N$ ha abierto tímidamente la puerta, y de forma paradójica mediante las redes sociales, a una relación más estrecha con los medios tradicionales

\section{Conclusiones}

Pese al rechazo inicial que suscitaron en el entorno de las relaciones públicas de las organizaciones políticas, la utilización de las redes sociales se ha ido normalizando, demostrando los beneficios que reporta. Así, tanto organizaciones políticas como sus dirigentes han descubierto, especialmente en Twitter, un nuevo instrumento para comunicar de manera descentralizada -sin que sea necesaria la intervención de los medios convencionales-, pero simultáneamente que sirva como catalizador de su fuerza y opinión. En consecuencia, cualquier agente sociopolítico que busque influir en la esfera pública ha comenzado a abrirse camino en el mundo digital.

Los populismos han demostrado su eficacia en la utilización de estas herramientas consiguiendo fidelizar el apoyo de la audiencia social, movilizarlo a las urnas y traducir los retweets en votos; tal y como hemos demostrado en este trabajo sobre el $R N$. En un primer momento la investigación se concentró en la mera descripción del uso que hacía el $R N$ de su cuenta oficial de Twitter. De esta manera, en 2014 nos encontrábamos con una comunicación monótona, poco atractiva y redundante. El mundo virtual no se había introducido de una forma tan trascendental en nuestra cotidianeidad y el $R N$ no supo manejar las redes correctamente y, por tanto, tampoco sacarles el rendimiento que pueden llegar a proporcionar. Por ello, la baja interacción entre el partido y sus seguidores no fue un indicio representativo del resultado de las urnas.

Sin embargo, cinco años más tarde las redes sociales se entienden como una extensión social del ser humano (Elías, 2015), por lo que una comunicación vía online eficaz resulta indispensable. Durante ese lapso temporal, el $R N$ supo ir abriéndose hueco:

- mejoró la apariencia de los tweets -en 2014, un 2\% contenía recursos audiovisuales frente al 78\% en 2019-;

- comenzó a mantener una conversación 2.0 en las redes (en 2014 el 98\% del contenido se dirigía a la propia web, mientras que en 2019 el usuario podía seguir el 95\% de la conversación en el propio perfil del $R N$ ).

Además, el "cordón sanitario" que le impusieron los medios tradicionales por ser de extrema derecha le hizo profundizar en la comunicación online como medida alternativa a la televisión, radio y prensa. El $R N$ acabó priorizando la difusión a través de las redes, porque conecta con la ciudadanía de forma directa pero fundamentalmente porque evita las críticas que el periodismo siempre ha vertido contra el sensacionalismo. Por ende, se puede confirmar su notable mejoría en las relaciones públicas a través de Twitter y por tanto se certifica la primera de nuestras hipótesis. En 2019, el $R N$ ha valorado su presencia en Twitter como un recurso principal de su estrategia.

Una vez verificada esta primera hipótesis, nos preguntamos por las fortalezas de la estrategia de comunicación online del $R N$ en la última campaña. La principal resultó ser la autoría o, mejor dicho, la transición de tener una voz (el perfil oficial) a considerar un abanico de miembros de la formación radical. La distribución del $60 \%$ de la muestra entre autores al margen de la cuenta oficial ha demostrado la visibilidad que el partido quiso otorgarles en la campaña del 2019, a través del retweeteo de sus publicaciones en el muro del partido. En consecuencia, el primer efecto de este cambio ha sido indudablemente el aumento en la interacción (el 80\% de los tweets en 2014 no contenían ninguna mención frente al 30\% en 2019) y en el nivel de implicación. Como hemos observado en esta investigación, en 2014 una mayoría de los mensajes no obtenían respuesta, mientras que en 2019 , hasta seis publicaciones llegaron a sobrepasar el millar de comentarios.

Esta retroalimentación originó una mayor propagación de su contenido, lo que se tradujo en que usuarios de Twitter, aún sin ser seguidores de la cuenta del $R N$, estuvieron expuestos a su mensaje electoral aumentando sus posibilidades de ser potenciales votantes del partido. De nuevo podemos certificar nuestra segunda hipótesis, pues ha sido gracias a una pluralidad de actores que el radio de alcance de su mensaje se ha expandido, provocando una mayor viralidad de sus contenidos.

Podemos afirmar que la nueva imagen cumple la expectativa de captatio benevolentiae, es decir, con su apariencia políticamente correcta consigue sumar sin asustar. De ahí que el $R N$ lograra conquistar de nuevo a más de un $23 \%$ del electorado francés en las elecciones europeas de mayo de 2019, reivindicando que a pesar de no complacer los requisitos del establishment, debía ser aceptado por la totalidad de la clase política como un rival más de la partida. El blanqueamiento de su imagen en redes sociales ha servido al $R N$ para acercar posiciones con una parte del electorado que, de no haber visto ese cambio de imagen en el $R N$ en estos últimos años, nunca habría considerado votar al partido de la hija del ultraderechista Le Pen. En resumen, la investigación ha demostrado que se trata de un ejemplo de éxito en relaciones públicas y comunicación organizacional online y redes sociales.

No obstante, nuestros resultados sugieren la importancia de abrir un debate teórico -que no podemos abordar aquísobre las estrategias de relaciones públicas de los populismos. Sería osado pretender que con el sólo análisis de una organización política podamos generalizar las hipótesis estudiadas para la mayoría de las formaciones populistas. En 
ese sentido, el análisis de contenido de los mensajes emitidos en Twitter por parte de la Lega en Italia, Fidesz en Hungría, el PiS en Polonia o incluso Vox en España, durante la última campaña electoral europea de 2019, constituiría una investigación de muestra ampliamente robusta, cuyos resultados podrían validar las hipótesis aquí expuestas como parte esencial de la estrategia de relaciones públicas del conjunto de organizaciones populistas. Igualmente, podría llevarse a cabo el mismo estudio en otra red social como es Facebook, con el objetivo de confirmar que su performance se repite en el conjunto de la comunicación online.

\section{Referencias}

Adams, Amelia; McCorkindale, Tina (2013). “Dialogue and transparency: A content analysis of how the 2012 presidential candidates used Twitter". Public relations review, v. 39, n. 4, pp. 357-359.

https://doi.org/10.1016/j.pubrev.2013.07.016

Alonso-Muñoz, Laura; Miquel-Segarra, Susana; Casero-Ripollés, Andreu (2016). "Un potencial comunicativo desaprovechado. Twitter como mecanismo generador de diálogo en campaña electoral”. Obra digital, v. 11, pp. 39-58. https://doi.org/10.25029/od.2016.100.11

Andrade, Horacio (2005). Comunicación organizacional interna: proceso, disciplina y técnica. Netbiblio. ISBN: 8497451007 https://bit.ly/2KGDIRB

Aruguete, Natalia (2017). "The agenda setting hypothesis in the new media environment". Comunicación y sociedad, n. 28, pp. 35-58.

https://doi.org/10.32870/cys.v0i28.2929

Barisione, Mauro; Michailidou, Asimina (eds.). (2017). Social media and European politics: Rethinking power and legitimacy in the digital era. Houndmills: Palgrave Macmillan. ISBN: 9781137598905

Bentivegna, Sara (2015). A colpi di tweet: la politica in prima persona. II Mulino. Bolonia ISBN: 9788815257215

Betz, Hans-George (2017). “The new politics of resentment. Radical right-wing populism in Western Europe”. In: Mudde, Cass (ed.). The populist radical right: A reader. New York: Routledge, pp. 338-351. ISBN: 9781138673878

Bimber, Bruce (2014). “Digital media in the Obama campaign of 2008 and 2012: Adaptation to the personalized political communication environment". Journal of information, technology \& politics, v. 11, n. 2, pp. 130-150.

https://doi.org/10.1080/19331681.2014.895691

Bouza-García, Luis; Tuñón-Navarro, Jorge (2018). “Personalización, distribución, impacto y recepción en Twitter del discurso de Macron ante el Parlamento Europeo el 17/04/18". El profesional de la información, v. 27, n. 6, pp. 1239-1247. https://doi.org/10.3145/epi.2018.nov.07

Bruns, Axel; Burgess, Jean (2011). "The use of Twitter hashtags in the formation of ad hoc publics". In: Proceedings of the $6^{\text {th }}$ European Consortium for Political Research (ECPR). General Conference 2011.

https://eprints.qut.edu.au/46515

Calvo, Dafne; Renedo-Farpón, Cristina; Díez-Garrido, María (2017). “Podemos in the regional elections 2015: Online campaign strategies in Castille and León". RIPS. Revista de investigaciones políticas y sociológicas, v. 16, n. 2, pp. 143-160. https://doi.org/10.15304/rips.16.2.3897

Campos-Domínguez, Eva (2017). "Twitter y la comunicación política". El profesional de la información, v. 26, n. 5, pp. 785-793.

https://doi.org/10.3145/epi.2017.sep.01

Canel, María-José; Sanders, Karen (2012). "Government communication: An emerging field in political communication research". In: Semetko, Holli A.; Scammell, Margaret (eds.). The SAGE handbook of political communication. London: SAGE, pp. 85-96. ISBN: 9781847874399

https://mariajosecanel.com/pdf/emergingfield.pdf

Carracedo, José-David (2002). "La vigilancia en las sociedades de la información ¿un panóptico electrónico?”. Política y sociedad, v. 39, n. 2, pp. 437-456.

https://revistas.ucm.es/index.php/POSO/article/view/POSO0202230437A

Casero-Ripollés, Andreu; Feenstra, Ramón A.; Tormey, Simón (2016). "Old and new media logics in an electoral campaign: The case of Podemos and the two-way street mediatization of politics". The international journal of press/politics, v. 21, n. 3, pp. 378-397.

https://doi.org/10.1177/1940161216645340 
Castells, Manuel (2009). Comunicación y poder. Madrid: Alianza. ISBN: 9788420684994

Castillo-Esparcia, Antonio; Guerra-Heredia, Sergio; Almansa-Martínez, Ana (2017). "Political communication and thinktanks in Spain. Strategies with the media”. El profesional de la información, v. 26, n. 4, pp. 706-713.

https://doi.org/10.3145/epi.2017.jul.14

Castillo-Esparcia, Antonio; Smolak-Lozano, Emilia; Fernández-Souto, Ana-Belén (2017). “Lobby y comunicación en España. Análisis de su presencia en los diarios de referencia”. Revista latina de comunicación social, n. 72, pp. 783-802. http://www.revistalatinacs.org/072paper/1192/RLCS-paper1192.pdf

Chadwick, Andrew (2006). Internet politics: States, citizens and new communication technologies. Oxford: Oxford University Press. ISBN: 9780195177732

Chadwick, Andrew (2013). The hybrid media system: Politics and power. Oxford: Oxford University Press. ISBN: 0199759480

Congosto, María-Luisa (2015). "Elecciones europeas 2014: Viralidad de los mensajes en Twitter". Redes. Revista hispana para el análisis de redes sociales, v. 26, n. 1.

https://doi.org/10.5565/rev/redes.529

Crépon, Sylvain; Dézé, Alexandre; Mayer, Nonna (2015). Les faux-semblants du Front National: Sociologie d'un parti politique. Paris: Presses de Sciences Po.

D’Adamo, Orlando; García-Beaudox, Virginia (2016). “Comunicación política: narración de historias, construcción de relatos políticos y persuasión". Comunicación y hombre, n. 12, pp. 23-39.

http://www.redalyc.org/articulo.oa?id=129446703001

Duggan, Maeve (2015). Mobile messaging and social media 2015. Pew Research Centre. http://www.pewinternet.org/2015/08/19/mobile-messaging-and-social-media-2015

Eiroa, Matilde; Barranquero, Alejandro (2017). Metodologías de investigación en la comunicación y sus medios. Madrid: Editorial Síntesis. ISBN: 9788491715757

Elías, Carlos (2015). El selfie de Galileo. Software social, político e intelectual del siglo XXI. Barcelona: Ediciones Península. ISBN: 9788499424248

Enli, Gunn S.; Skogerbø, Eli (2013). "Personalized campaigns in party-centred politics: Twitter and Facebook as arenas for political communication". Information, communication \& society, v. 16, n. 5, pp. 757-774.

https://doi.org/10.1080/1369118X.2013.782330

Eom, Young-Ho; Puliga, Michelangelo; Smailović, Jasmina; Mozetič, Igor; Caldarelli, Guido (2015). "Twitter-based analysis of the dynamics of collective attention to political parties". Plos one, v. 10, n. 7.

https://doi.org/10.1371/journal.pone.0131184

Fernández-Vázquez, Guillermo (2019). ¿Qué hacer con la extrema derecha en Europa? El caso del Frente Nacional. Madrid: Lengua de Trapo. ISBN: 9788483812389

Fielding, Nigel G.; Lee, Raymond M.; Blank, Grant (eds.) (2008). The handbook of online research methods. London: SAGE. ISBN: 9781473918788

Gainous, Janson; Wagner, Kevin M. (2014). Tweeting to power: The social media revolution in American politics. Oxford University Press. ISBN: 9780199965076

https://doi.org/10.1093/acprof:oso/9780199965076.001.0001

Galeano, Susana (2019). “Cuáles son las redes sociales con más usuarios del mundo (2019)”. Marketing for ecommerce. https://bit.ly/2Py5dAt

García-Orosa, Berta (2009). Gabinetes de comunicación online: claves para generar información corporativa. Sevilla: Comunicación Social. ISBN: 9788496082786

García-Orosa, Berta; Vázquez-Sande, Pablo; López-García, Xosé (2017). “Narrativas digitales de los principales partidos políticos de España, Francia, Portugal y Estados Unidos". El profesional de la información, v. 26, n. 4, pp. 589-600. https://doi.org/10.3145/epi.2017.jul.03

Giglietto, Fabio; Selva, Donatella (2014). "Second screen and participation: A content analysis on a full season dataset of tweets". Journal of communication, v. 64, n. 2, pp. 260-277

Golbeck, Jennifer; Grimes, Justin M.; Rogers, Anthony (2010). "Twitter use by the U.S. Congress". Journal of the American Society for Information Science and Technology, v. 61, n. 8, pp. 1612-1621.

https://doi.org/10.1002/asi.21344 
Graham, Todd; Jackson, Dan; Broersma, Marcel (2016). “New platform, old habits? Candidates' use of Twitter during the 2010 British and Dutch general elections campaigns". New media and society, v. 18, n. 5, pp. 765-783.

https://doi.org/10.1177/1461444814546728

Grunig, Larissa A.; Grunig, James E.; Dozier, David M. (2003). Excellent public relations and effective organizations: A study of communication management in three countries. New York: Routledge. ISBN: 9780805818185 https://bit.ly/2W5IKMW

Guerrero-Pico, Mar; Scolari, Carlos A. (2016). "Narrativas transmedia y contenidos generados por los usuarios: el caso de los cross-overs". Cuadernos.info, n. 8, pp. 183-200.

https://doi.org/10.7764/cdi.38.760

Gutiérrez-Rubí, Antoni (2014). Tecnopolítica. Madrid: Bebookness. ISBN: 9788469718063

http://www.gutierrez-rubi.es/tecnopolitica

Habermas, Jürgen (2017). “¿Qué nos costará esta vez a los alemanes?”. El país, 10 diciembre. https://elpais.com/elpais/2017/12/08/opinion/1512750567_704832.html

Hine, Christine (2004). Etnografía virtual. Barcelona: UOC. ISBN: 8497880196

Hine, Christine (2005). Virtual methods: Issues in social research on the Internet. Oxford: Berg Publishers. ISBN: 9781 845200848

Jackson, Nigel; Lilleker, Darren (2011). “Microblogging, constituency service and impression management: UK MPs and the use of Twitter". The journal of legislative studies, v. 17, n. 1, pp. 86-105.

https://doi.org/10.1080/13572334.2011.545181

Jivkova-Semova, Dimitrina; Requeijo-Rey, Paula; Padilla-Castillo, Graciela (2017). "Usos y tendencias de Twitter en la campaña a elecciones generales españolas del 20D de 2015: hashtags que fueron trending topic". El profesional de la información, v. 26, n. 5, pp. 824-837.

https://doi.org/10.3145/epi.2017.sep.05

Jones, Steve (1998). Doing internet research. Critical issues and methods for examining the net. London: SAGE. ISBN: 978 0761915959

Jungherr, Andreas (2014). Twitter in politics: a comprehensive literature review.

https://ssrn.com/abstract=2402443

Jungherr, Andreas; Schoen, Harald; Jürgens, Pascal (2015). "The mediation of politics through Twitter: An analysis of messages posted during the campaign for the German federal election 2013". Journal of computer-mediated communication, v. 21, n. 1, pp. 50-68.

https://doi.org/10.1111/jcc4.12143

Kozinets, Robert (2010). Netnography: Doing ethnographic research online. London: SAGE. ISBN: 9781848606456

Kreiss, Daniel (2016). "Seizing the moment: The presidential campaign's use of Twitter during the 2012 electoral cycle". New media \& society, v. 18, n. 8, pp. 1473-1490.

https://doi.org/10.1177/1461444814562445

Larrondo-Ureta, Ainara (2016). “Comunicación organizacional ante el reto de la estrategia multiplataforma y 2.0: la experiencia de los partidos políticos en el País Vasco". El profesional de la información, v. 25, n. 1, pp. 114-123.

https://doi.org/10.3145/epi.2016.ene.11

Larsson, Anders-Olof (2015). "The EU Parliament on Twitter - Assessing the permanent online practices of parliamentarians". Journal of information technology \& politics, v. 12, n. 2, pp. 149-166.

https://doi.org/10.1080/19331681.2014.994158

Larsson, Anders; Kalsnes, Bente (2014). "'Of course we are on Facebook': Use and non-use of social media among Swedish and Norwegian politicians". European journal of communication, v. 29, n. 6, pp. 653-667.

https://doi.org/10.1177/0267323114531383

Lévy, Pierre (2004). Ciberdemocracia. Ensayo sobre filosofía política. Barcelona: Editorial UOC. ISBN: 9788497881333

López-Meri, Amparo; Marcos-García, Silvia; Casero-Ripollés, Andreu (2017). "What do politicians do on Twitter? Functions and communication strategies in the Spanish electoral campaign of 2016". El profesional de la información, v. 26, n. 5, pp. 795-804.

https://doi.org/10.3145/epi.2017.sep.02

Maarek, Philippe J. (2011). Campaign communication and political marketing. New York: John Wiley \& Sons. ISBN: 978 1444332353 
Maarek, Philippe J. (2014). "Politics 2.0: New forms of digital political marketing and political communication". Trípodos, n. 34, pp. 13-22.

http://www.tripodos.com/index.php/Facultat_Comunicacio_Blanquerna/article/view/163

Marcos-García, Silvia (2018). Las redes sociales como herramienta de la comunicación política. Usos políticos y ciudadanos de Twitter e Instagram. Tesis doctoral, Universitat Jaume I.

https://www.tdx.cat/handle/10803/662817

Mazzoleni, Gianpietro (2001). “La revolución simbólica de Internet”. Cuadernos de información y comunicación, n. 6, pp. 33-39. https://revistas.ucm.es/index.php/CIYC/article/view/CIYC0101110033A

Mosca, Lorenzo; Vaccari, Cristian (2011). Nuovi media, nuova política. Partecipazione e mobilitazione da MoveOn al Movimento 5 Stelle. Milan: Franco Angeli. ISBN: 9788856840315

Papagianneas, Stavros (2017). Rebranding Europe. Fundamentals for leadership communication. Brussels: ASP editions. ISBN: 9789057186202

Parmelee, John H.; Bichard, Shanoon L. (2012). Politics and the Twitter revolution. How tweets influence the relationship between political leaders and the public. Lanham, MD. Lexington Books. ISBN: 0739165011

Peñafiel, Carmen (2016). "Reinvención del periodismo en el ecosistema digital y narrativas transmedia". AdComunica, n. 12 , pp. 163-183.

http://repositori.uji.es/xmlui/handle/10234/167719

Quan-Haase, Anabel; Sloan, Luke (2016). "Introduction to the Handbook of social media research methods: Goals, Challenges and Innovations". In: Sloan, Luke; Quan-Haase, Anabel. The SAGE hankbook of social media research methods. Londres. SAGE. ISBN: 9781473916326

https://doi.org/10.4135/9781473983847.n1

Rodríguez-Andrés, Roberto; Ureña-Uceda, Daniel (2011). "Diez razones para el uso de Twitter como herramienta de comunicación política y electoral". Comunicación y pluralismo, v. 10, pp. 89-115.

https://core.ac.uk/download/pdf/50605323.pdf

Salaverría, Ramón (2015). “Los labs como fórmula de innovación en los medios”. El profesional de la información, v. 24, n. 4, pp. 397-404. https://doi.org/10.3145/epi.2015.jul.06

Sanjuán-Santonja, Mar (2015). “Nuevas formaciones políticas crecidas en Internet y su entrada en las elecciones europeas 2014: el caso de Movimiento RED, Podemos, Recortes Cero y Partido X”. Dígitos, v. 1, pp. 71-90.

https://revistadigitos.com/index.php/digitos/article/view/4

Scherpereel, John A.; Wohlgemuth, Jerry; Schmelzinger, Margaret (2016). "The adoption and use of Twitter as a re-presentational tool among members of the European Parliament". European politics and society, v. 18, n. 2, pp. 111-127. https://doi.org/10.1080/23745118.2016.1151125

Schoeneborn, Dennis (2011). “Organization as communication: A Luhmmanian perspective". Management organization quarterly, v. 25, n. 4, pp. 663-689.

https://doi.org/10.1177/0893318911405622

Scolari, Carlos A.; Fernández-de-Azcárate, Sara; Garín, Manuel; Guerrero, Mar; Jiménez, Manel; Martos, Aitor; Obradors, Matilde; Oliva, Mercè; Pérez, Óliver; Pujadas, Eva (2012). “Narrativas transmediáticas, convergencia audiovisual y nuevas estrategias de comunicación”. Quaderns del CAC, v. 15, n. 1, pp. 79-89.

https://www.cac.cat/sites/default/files/2019-01/Q38_scolari_et_al_ES.pdf

Serrano-Cobos, Jorge (2016). "Tendencias tecnológicas en internet: hacia un cambio de paradigma". El profesional de la información, v. 25, n. 6, pp. 843-850.

https://doi.org/10.3145/epi.2016.nov.01

Spitulnik-Vidali, Debra; Peterson, Mark (2012). "Ethnography as theory and method in the study of political communication". In: Semetko, Holli A.; Scammell, Margaret (eds.). The SAGE handbook of political communication. London: SAGE, pp. 264-275. ISBN: 9781847874399

Steward, Bonnie (2017). "Twitter as method: Using Twitter as a tool to conduct research". In: Sloan, Luke; Quan-Haase, Anabel. The SAGE hankbook of social media research methods. Londres. SAGE. ISBN: 9781473916326 https://doi.org/10.4135/9781473983847.n16

Stromer-Galley, Jennifer (2014). Presidential campaigning in the Internet age. Oxford: Oxford University Press. ISBN: 9780199731947

Túñez-López, Miguel (2012). La gestión de la comunicación en las organizaciones. Sevilla: Comunicación Social Ediciones y Publicaciones. ISBN: 9788492860937 
Tuñón-Navarro, Jorge (2017). Comunicación internacional. Información y desinformación global en el siglo XXI. Madrid: Fragua. ISBN: 9788470747472

Tuñón-Navarro, Jorge; Bouza-García, Luis; Carral, Uxía (2019). Comunicación Europea ¿A quién doy like para hablar con Europa? Madrid: Dykinson. ISBN: 9788491489771

Tuñón-Navarro, Jorge; Carral, Uxía (2019). “Twitter como solución a la comunicación europea. Análisis comparado en Alemania, Reino Unido y España". Revista latina de comunicación social, v. 74, pp. 1219-1234. https://doi.org/10.4185/RLCS-2019-1380

Tuñón-Navarro, Jorge; Catalán-Matamoros, Daniel (2020). “Comparación de las estrategias de campaña online para albergar la Agencia Europea de Medicamentos". El profesional de la información, v. 29, n. 2, e290225. https://doi.org/10.3145/epi.2020.mar.25

Vergeer, Maurice; Hermans, Liesbeth (2013). "Campaigning on Twitter: Microblogging and online social networking as campaign tools in the 2010 general elections in the Netherlands". Journal of computer-mediated communication, v. 18, n. 4, pp. 399-419.

https://doi.org/10.1111/jcc4.12023

Veerger, Maurice; Hermans, Liesbeth; Sams, Stevens (2013). "Online social networks and micro-blogging in political campaigning: The exploration of a new campaign tool and a new campaign style". Party politics, v. 19, n. 3, pp. 477-501. https://doi.org/10.1080/1354068811407580

\section{Anexo}

Tabla 1. Ficha de análisis de contenido

Nivel de narración

\begin{tabular}{|l|l|l|}
\hline \multicolumn{1}{|c|}{$\begin{array}{c}\text { 1. Temática } \\
\text { ¿Sobre qué tweetean? }\end{array}$} & \multicolumn{1}{c|}{$\begin{array}{c}\text { 2. Autoría } \\
\text { ¿Quién emite contenido? }\end{array}$} & $\begin{array}{c}\text { 3. Finalidad } \\
\text { ¿Para qué tweetean? }\end{array}$ \\
\hline 1. Inmigración/Seguridad/Fronteras & 1. Propia cuenta de RN/FN & 1. Propaganda de sus encuentros \\
\hline 2. Política exterior (UE y Brexit) & 2. Marine Le Pen & 2. Informar de su programa \\
\hline 3. Economía/empleo/energía & 3. Jordan Bardella & 3. Promocionar aparición en medios tradicionales \\
\hline $\begin{array}{l}\text { 4. Política social (educación, sanidad, eutana- } \\
\text { sia, feminismo, etc.) }\end{array}$ & 4. Miembro político del RN/aliados & 4. Confrontar a los rivales políticos o mediáticos \\
\hline 5. Justicia & 5. Medio de comunicación & 5. Emitir una queja \\
\hline 6. Campaña electoral/partido & 6. Actores adversarios & 6. Movilizar el voto \\
\hline 7. Otros (clima, agricultura, cultura, identidad) & 7. Otros & 7. Exaltación emotiva, patriotismo \\
\hline
\end{tabular}

Nivel de interacción

\begin{tabular}{|l|l|}
\multicolumn{1}{|c|}{ 4. Participación } \\
¿A quién se hace partícipe o apela? & \multicolumn{1}{c}{$\begin{array}{c}\text { 5. Contenido } \\
\text { ¿Qué vertebra la conversación? }\end{array}$} \\
\hline 1. Tweet sin mención & 1. Enlace a su propia web/redes \\
\hline 2. Tweet con @\# mención a miembro RN/aliado & 2. Enlace a un medio \\
\hline 3. Tweet con @\# mención a rival & 3. Fotografía \\
\hline 4. Tweet con @\# mención a un medio & 4. Vídeo / audio \\
\hline 5. Tweet con @\# mención a otros (instituciones) & 5. Texto solamente (u otro tweet) \\
\hline
\end{tabular}

Nivel de implicación

\begin{tabular}{|c|c|c|}
\hline $\begin{array}{c}\text { 6. Compartir } \\
\text { ¿Cuántos RTs ha conseguido? }\end{array}$ & $\begin{array}{c}\text { 7. Gustar } \\
\text { ¿Cuántos favs ha conseguido? }\end{array}$ & $\begin{array}{c}\text { 8. Comentar } \\
\text { ¿Cuántos han respondido? }\end{array}$ \\
\hline 1. 1-5 RTs & 1. 1-5 favs & 1. 1-5 comentarios \\
\hline 2. 6-25 RTs & 2. 6-25 favs & 2. 6- 5 comentarios \\
\hline 3. $26-100 \mathrm{RTs}$ & 3. $26-100$ favs & 3. 25-100 comentarios \\
\hline 4. $101-200 \mathrm{RTs}$ & 4. 101-200 favs & 4. 101-200 comentarios \\
\hline 5. $201-300 \mathrm{RTs}$ & 5. 201-300 favs & 5. 201-300 comentarios \\
\hline 6. 301-400 RTs & 6. 301-400 favs & 6. $301-400$ comentarios \\
\hline 7. 401-500 RTs & 7. 401-500 favs & 7. 401-500 comentarios \\
\hline 8. Más de 500 RTs & 8. Más de 500 favs & 8. Más de 500 comentarios \\
\hline 9. 0 RTs & 9. 0 favs & 9.0 comentarios \\
\hline
\end{tabular}

